\title{
An Enhanced Method of Secured Energy - Efficient Data Communication in MANET for Emergency Rescue Operation
}

\author{
Jayachitra. S, Thiagarajan. $\mathbf{N}$
}

\begin{abstract}
Mobile Adhoc Network (MANET) is a self configuring network consists of number of mobile nodes which can move in any direction at any time. The MANET is implemented for providing several applications such as border security, emergency rescue operation and business meetings, etc. Data communication between the mobile nodes in wireless environment mainly depends on life time of battery and storage capacity of mobile nodes. This paper presents about design of routing algorithms in order to provide data communication not only energy efficient but also secured. Three methods named Energy Optimized Node Partitioned Cluster Routing (EONPCR), Energy - efficient Polynomial Time Resource Channelized (EE-PTRC) framework and Secured QoS routing and Data Delivery method are proposed. The EO-NPCR method reduces routing delay by identifying appropriate neighbourhood nodes using Neighbourhood Sector-based energy conservation model. The EE-PTRC framework uses Two Hop Propagate Bandwidth Allocation model for reducing the bandwidth consumption also it improves data delivery. The third method named Secured QoS Routing and Data Delivery (SQR-DD) framework provides data communication over secured path also satisfying the QoS, it applies Geo-optimized maximum likelihood routing. The result of three methods is to achieve secured data communication in emergency rescue application by enhancing energy - efficient routing.
\end{abstract}

Keywords: MANET; EO-NPCR; EE-PTRC; SQR-DD.

\section{INTRODUCTION}

Mobile Ad Hoc network (MANET) is an infrastructureless network, which is constructed with the number of mobile nodes. Data communication among mobile nodes is achieved wirelessly to convey the data from source to destination node. Due to the limited battery and storage capacity in mobile nodes energy - efficient routing is essential to minimize the cost of energy during data communication. Several routing algorithms are designed to improve energy efficiency, reliability and lifetime. These routing algorithms do not improve the secured QoS routing and efficient data delivery of the data communication system. To enhance the data communication in a secured environment and provide energy - efficient routing the following methods are proposed.

Revised Manuscript Received on December 12, 2019

Jayachitra. S, Associate Professor, Department of Electronics and Communication Engineering, Vivekanandha College of Engineering For Women, Tiruchengode, Tamil Nadu 637205, India

Thiagarajan. N, Assistant Professor, Department of Medical Electronics, Sengunthar College of Engineering, Tiruchengode, Tamil Nadu, 637205, India
To begin with, Energy Optimized Node Partitioned Cluster Routing (EO-NPCR) method is developed for providing the efficient energy route discovery. Effective emergency rescue operation is developed using with low routing overhead for data transmission. In EO-NPCR the routing delay on dynamically changing topology is reduced and that identifies the neighbouring nodes by using Neighbourhood Sector-based energy conservation model. Next, Partitioned Cluster Routing is implemented for minimizing the node energy drain rate using mobility factor and Friis transmission in the EO-NPCR method. The number of movable mobile users (member) which form a similar type of route path are clustered together to make a hierarchical control mobile network environment. The mobile users utilize the clustered group for broadcasting the information about the rescue area in a connected path. The connected-path link dominant set consist of the cluster head (mobile information collector) pruning rules to remove the repetition (i.e. unauthorized members).

In addition, the partitioned cluster routing algorithm is employed to enhance the cluster accuracy by choosing the optimal cluster head. At the Last, the Geo-Optimized Maximum Likelihood function optimizes the route discovery to minimize the energy consumption rate, which helps in providing emergency rescue operation. Moreover, the removal of repetition reduces the routing overhead to ensure data communication between groups of rescuer and preserves the data reliability.

Then, Energy - efficient Polynomial Time Resource Channelized (EE-PTRC) framework is developed with the objective of improving the data communication for providing emergency rescue operation. Initially, the EEPTRC framework is introduced for improving the data communication on multiple mobile units in message broadcasting between the rescuers. Next, the Two Hop Propagate Bandwidth Allocation model in the EE-PTRC framework is integrated to reduce the bandwidth consumption and improves the data delivery rate. Bandwidth allocation of the intermediate mobile nodes is estimated with traffic condition that is mainly based on the channels and the load balancing capacity of the Geooptimized route path. By estimating the channel based on the weight, and through the channel the mobile nodes are used to communicate with each other based on minimum channel weight. Finally, the Polynomial Time Resource Conserved Channelized approach maximizes the energy efficiency. 
This in turn reduces the data delivery overhead by applying the cost function with respect to the time interval.

In the end, an efficient method called Secured QoS Routing and Data Delivery (SQR-DD) framework is designed for improving the data communication and provides better quality of service in a secured environment. The proposed SQR-DD framework provides secure emergency rescue operations. The movable mobile unit (members) starts by searching the entire shortest route path and picks up the minimal energy consumption path in mobile ad-hoc network for sharing the information. In addition, the Mobile Information Collector (MIC) also acts as a gateway to the rescue operation center that provides information about the area. This helps to select the minimal energy conservation path for information sharing. The QoS is provided by examining user (mobile nodes) requested data delivery time to resource efficient Geo-optimized maximum likelihood routing. Node cooperativeness factor is accessed for every mobile node in the network. Ultimately, enhanced cooperative intermediate nodes in the routing paths are established to provide QoS for securing user requested services and efficiently utilizing the resources. Therefore, secure data communication is achieved in emergency rescue application and energy - efficient routing is also enhanced. Fig. 1. Explains the architecture of emergency rescue application using proposed methods.

In order to initiate a rescue operation, a temporary network communication and information communications are made in the affected area. Rescuers need to discuss between group members and to organize the rescue operation.

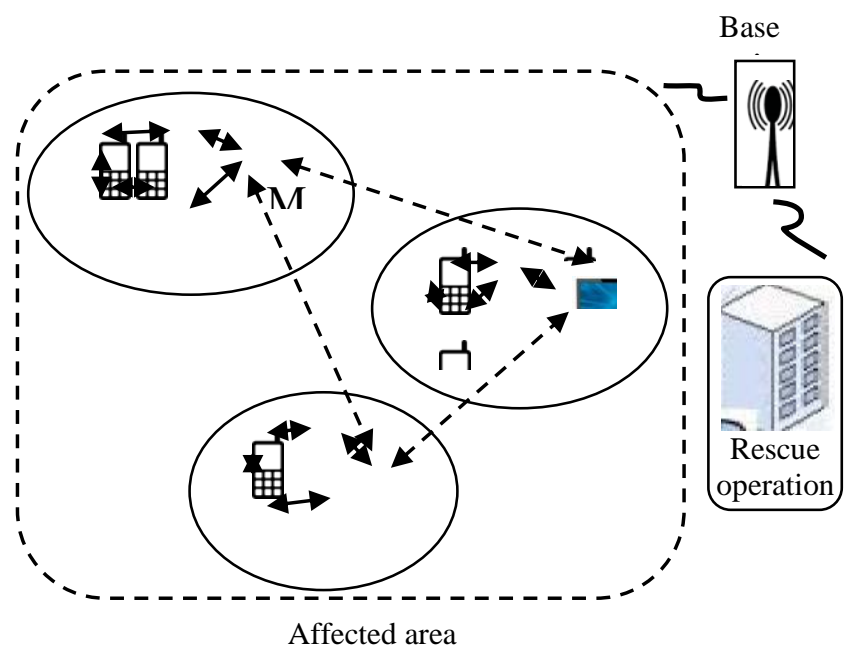

Fig. 1 MANET with emergency rescue operation

All the information required to be communicated from the teams and the rescue operation center and conversely in order for the rescue work and save lives. By using a small mobile device, the information is transmitted from one rescue group member to another group member. The Mobile unit transmits networking tools to support routing operations. A no. of mobile devices are connected with the Mobile Information Collector (MIC) (i.e. Leader) which is in a stationary condition. In rescue operations, the rescue team shares the information between the group members in the affected location in a secured manner. Each team member has the unique controller to distribute their information. This information is distributed to a rescue operation center by way of the mobile base station. In order to improve the emergency rescue operation, three different methods are significantly developed to analyze the efficient route authentication for secured information sharing. While performing these communications, proposed EO-NPCR, EE-PTRC and SQR-DD methods are utilized to identify the energy - efficient route. Therefore, secure communications are achieved in effective emergency rescue application.

\section{PERFORMANCE ANALYSIS FOR PROPOSEDEO-NPCR, EE-PTRC AND SQR-DD METHODS}

Experiments are conducted using NS2 simulator for proposed EO-NPCR, EE-PTRC and SQR-DD methods and existing methods, namely Distributed Cache Invalidation Method (DCIM) developed by KassemFawaz\& Hassan Artail (2013) and namely Neighbour Coverage-based Probabilistic Rebroadcast (NC-PR) method developed by Xin Ming Zhang et al. (2013). A network is constructed in the square area of $1500 \mathrm{~m} * 1500 \mathrm{~m}$ with 100 mobile nodes that uses Random Way Point (RWM) model. To conduct the experiment number of data packets is taken as 7 and maximum size of data packets is taken as $70 \mathrm{~KB}$. Node speed is varied from 0 to $10 \mathrm{~m} / \mathrm{s}$. The simulation time is 50 milliseconds. Destination Sequence based Distance Vector (DSDV) protocol is used on proposed methods for performing the simulations.

The measurement of the factors in terms of routing energy consumption routing delay, node energy drain rate, clustering accuracy, routing energy consumption, data delivery rate, data delivery overhead, bandwidth utilization 
for rendering quality of service are carried out using NS-2 simulations. The result analyses of these metrics are explained in further section. The simulation results are compared and analyzed with the help of the table and graph.

\section{A. Impact of routing energy consumption}

The routing energy consumption is calculated as the amount of energy required for data packet transmission between the source mobile node and receiver mobile nodes. The routing energy consumption is measured in terms of
Joules $(\mathbf{J})$.The routing energy consumption is calculated using the formula as follows.

$E C_{R}=D P_{i} *$ Energyrequired for each data packet

In the equation (1) the routing energy consumption is denoted as ' $E C_{R}$ ' that is obtained using the data packets ' $D P_{i}$ ' and energy required for each data packet. If the routing energy consumption is less, then the method is said to be more efficient.

Table. 1 Tabulation for routing energy consumption

\begin{tabular}{|c|c|c|c|c|c|}
\hline \multirow{2}{*}{$\begin{array}{c}\text { Number of data } \\
\text { packets }\end{array}$} & $\begin{array}{c}\text { Existing } \\
\text { DCIM }\end{array}$ & $\begin{array}{c}\text { Existing } \\
\text { NC-PR }\end{array}$ & $\begin{array}{c}\text { Proposed } \\
\text { EO-NPCR }\end{array}$ & $\begin{array}{c}\text { Proposed } \\
\text { EE-PTRC }\end{array}$ & $\begin{array}{c}\text { Proposed } \\
\text { SQR-DD }\end{array}$ \\
\hline 7 & 52.42 & 40.12 & 23.15 & 34.05 & 27.31 \\
\hline 14 & 63.21 & 53.33 & 35.81 & 47.89 & 41.22 \\
\hline 21 & 75.22 & 70.32 & 51.38 & 63.46 & 56.31 \\
\hline 28 & 71.54 & 66.52 & 49.21 & 61.29 & 53.42 \\
\hline 35 & 90.33 & 85.22 & 68.33 & 79.42 & 72.56 \\
\hline 42 & 85.36 & 78.34 & 62.19 & 72.27 & 66.23 \\
\hline 49 & 96.55 & 90.12 & 75.20 & 87.28 & 79.52 \\
\hline 56 & 102.32 & 96.15 & 79.14 & 90.47 & 84.15 \\
\hline 63 & 110.12 & 100.36 & 85.26 & 93.23 & 89.35 \\
\hline 70 & 115.24 & 104.66 & 89.21 & 98.13 & 93.53 \\
\hline
\end{tabular}

Comparison of routing energy consumption while data packet transmission for proposed EO-NPCR, EE-PTRC, SQR-DD methods and existing DCIM, NC-PR methods are shown in Table1. Number of data packets is taken from the range of 7 to 70 for conducting experiment. Table 1 clearly shows that, for the increase in the number of data packets, routing energy consumption is also increased for all methods. But in the EO-NPCR, routing energy consumption is significantly reduced when compared to other methods.

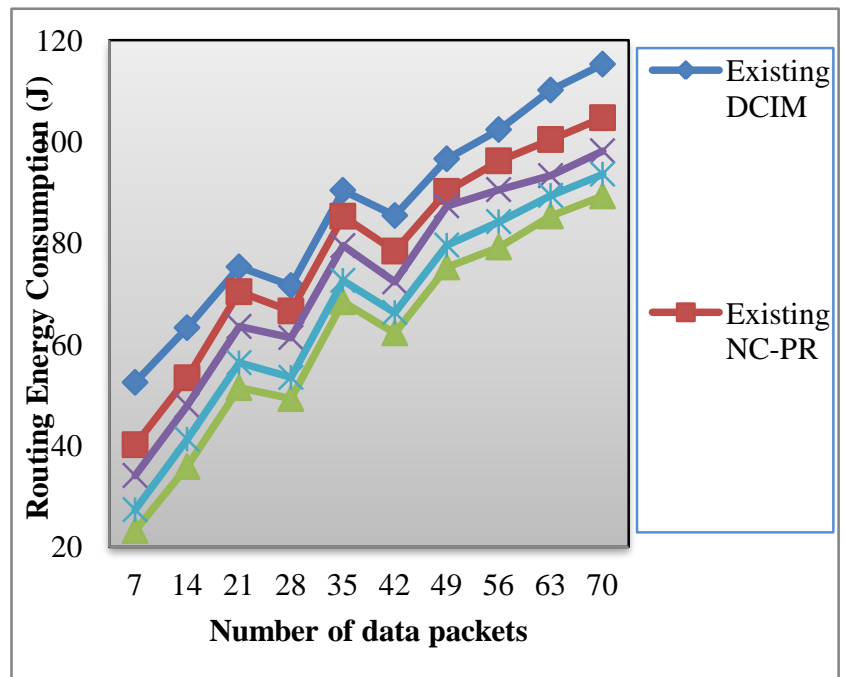

Fig. 2 Measure of routing energy consumption

Fig. 2 shows the comparison of routing energy consumption using different methods such as EO-NPCR, EE-PTRC, SQR-DD and existing DCIM method and NCPR method. From Fig. 2, it is clear that the EO-NPCR considerably reduces the routing energy consumption compared to other proposed and existing methods. This reduction of routing energy consumption is achieved in the proposed method by using Geo-Optimized Maximum Likelihood (GOML) function in emergency rescue operation. With the help of GOML function, the maximum likelihood path is efficiently discovered which resulting in reduced routing energy consumption. Therefore, routing energy consumption using EO-NPCR is reduced by $37 \%$ when compared to existing DCIM and NC-PR methods. Similarly, SQR-DD method reduces routing energy consumption by $29 \%$ and EE-PTRC framework reduces routing energy consumption by $15 \%$ compared to existing DCIM and NC-PR methods.

\section{B. Impact of Clustering Accuracy}

The clustering accuracy is calculated as the relation between number of properly clustered node to the number of mobile node density through the access points. Clustering accuracy is measured in terms of percentage $(\%)$ as given below.

$C A=\left(\frac{\text { Nodes properly clustered }}{M N D}\right) * 100$

In the equation (2), the ' $C A$ ' is denoted as clustering accuracy which is calculated with respect to mobile node density ' $M N D$ '. Higher the nodes being properly clustered, then the clustering accuracy is said to be more efficient.

Table 2 shows the comparison for clustering accuracy in terms of the number of mobile nodes using proposed 
EO-NPCR, EE-PTRC, SQR-DD methods and existing DCIM, NC-PR methods. The Number of mobile node density is taken from the range of 10 to 100 for conducting experiments.
Table 2 clearly shows that, for the increasing number of mobile node density, clustering accuracy is also increased for all methods. But in EO-NPCR clustering accuracy is significantly increased compared to other methods.

Table. 2 Tabulation for clustering accuracy

\begin{tabular}{|c|c|c|c|c|c|}
\hline \multirow{2}{*}{\begin{tabular}{c} 
Mobile Node $\begin{array}{c}|c| \\
\text { Density }\end{array}$ \\
\cline { 2 - 6 }
\end{tabular}} & $\begin{array}{c}\text { Existing } \\
\text { DCIM }\end{array}$ & $\begin{array}{c}\text { Existing } \\
\text { NC-PR }\end{array}$ & $\begin{array}{c}\text { Proposed } \\
\text { EO-NPCR }\end{array}$ & $\begin{array}{c}\text { Proposed } \\
\text { EE-PTRC }\end{array}$ & $\begin{array}{c}\text { Proposed } \\
\text { SQR-DD }\end{array}$ \\
\hline 10 & 31.12 & 37.12 & 62.35 & 44.35 & 51.83 \\
\hline 20 & 39.23 & 46.22 & 65.83 & 53.70 & 58.80 \\
\hline 30 & 45.38 & 52.56 & 71.29 & 59.16 & 64.26 \\
\hline 40 & 41.33 & 48.12 & 68.25 & 55.12 & 61.22 \\
\hline 50 & 48.48 & 55.62 & 74.33 & 63.20 & 68.30 \\
\hline 60 & 50.11 & 57.11 & 77.89 & 64.76 & 69.86 \\
\hline 70 & 53.44 & 60.33 & 81.35 & 68.22 & 73.32 \\
\hline 80 & 55.45 & 62.12 & 84.30 & 70.26 & 75.11 \\
\hline 90 & 57.22 & 64.33 & 86.12 & 72.49 & 77.52 \\
\hline 100 & 59.64 & 66.45 & 88.46 & 74.30 & 80.22 \\
\hline
\end{tabular}

The comparison of clustering accuracy, using different methods such as proposed EO-NPCR, EE-PTRC, SQR-DD and existing DCIM method and NC-PR method is shown in Fig. 3. The Fig. 3 also shows that the clustering accuracy is more for EO-NPCR compared to other proposed and existing methods. This efficient improvement in cluster accuracy is achieved in the by applying, the application of Partitioned Cluster Routing (PCR) algorithm in emergency rescue operation. The PCR algorithm discovers the cluster head in different rectangular regions that is mainly based upon mobility and mean node energy drain rate in an optimized manner.

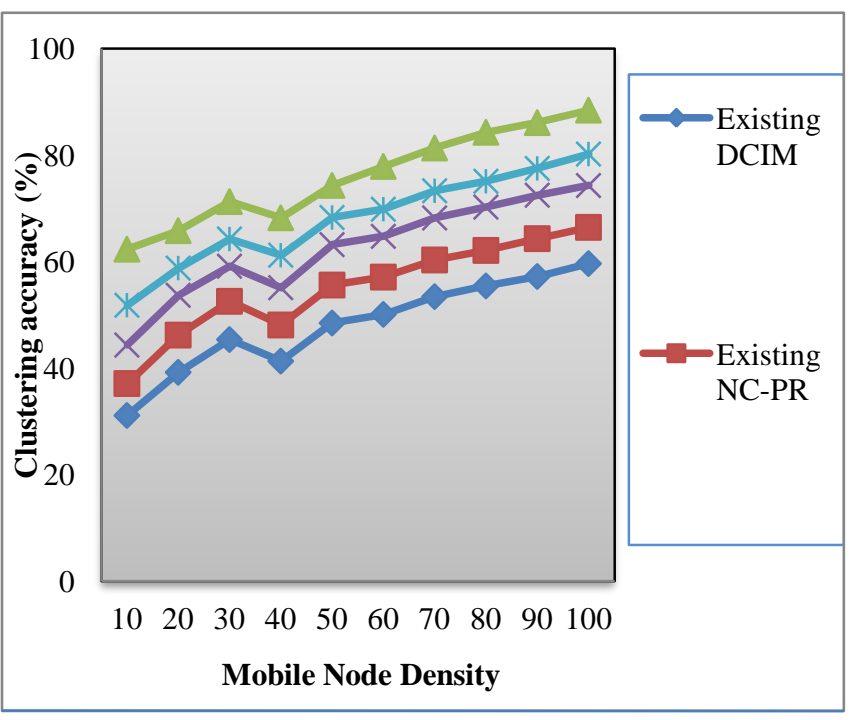

Fig. 3 Measure of clustering accuracy

Hence, clustering accuracy, using EO-NPCR is increased by $37 \%$ when compared to existing DCIM and NC-PR methods. Similarly, SQR-DD method increases clustering accuracy by $28 \%$ and EE-PTRC framework increases clustering accuracy by $19 \%$ compared to existing DCIM and NC-PR methods.

\section{Impact of Data Delivery Rate}

The data delivery rate is measured by the ratio of received data packets at destination mobile node and total number of data packets sent by the source mobile node. The data delivery rate is calculated as shown below.

$D D R=\frac{\text { received data packets }}{\text { total number of data packets sent }} * 100$

In the equation (3), the data delivery rate ' $D D R$ ' is measured in terms of percentage $(\%)$. If the data delivery rate gets higher, then the method is said to be more efficient. 
Table. 3 Tabulation for Data Delivery Rate

\begin{tabular}{|c|c|c|c|c|c|}
\hline \multirow{2}{*}{ Data Packets } & \multicolumn{5}{|c|}{ Data Delivery Rate (\%) } \\
\cline { 2 - 6 } & $\begin{array}{c}\text { Existing } \\
\text { DCIM }\end{array}$ & $\begin{array}{c}\text { Existing } \\
\text { NC-PR }\end{array}$ & $\begin{array}{c}\text { Proposed } \\
\text { EO-NPCR }\end{array}$ & $\begin{array}{c}\text { Proposed } \\
\text { EE-PTRC }\end{array}$ & $\begin{array}{c}\text { Proposed } \\
\text { SQR-DD }\end{array}$ \\
\hline 7 & 49.22 & 56.63 & 64.29 & 72.12 & 77.39 \\
\hline 14 & 52.62 & 59.12 & 67.35 & 73.25 & 80.45 \\
\hline 21 & 50.44 & 57.33 & 65.25 & 75.12 & 79.21 \\
\hline 28 & 48.22 & 55.21 & 63.21 & 72.11 & 81.35 \\
\hline 35 & 50.34 & 57.22 & 65.25 & 74.79 & 84.99 \\
\hline 42 & 52.41 & 59.42 & 67.89 & 77.10 & 87.16 \\
\hline 49 & 54.89 & 61.52 & 70.21 & 80.15 & 90.15 \\
\hline 56 & 56.92 & 64.22 & 73.25 & 83.69 & 93.16 \\
\hline 63 & 58.73 & 66.52 & 74.89 & 87.16 & 96.58 \\
\hline
\end{tabular}

Table 3 compares the data delivery rate with respect to the number of data packets of proposed EO-NPCR, EEPTRC, SQR-DD methods with existing DCIM, NC-PR methods. The Number of data packet is taken from the range of 7 to 70 , this table also shows that, for increasing in the number of data packet, data delivery rate are also increased for all methods. However, the SQR-DD method significantly improves the data delivery rate compared to other methods.

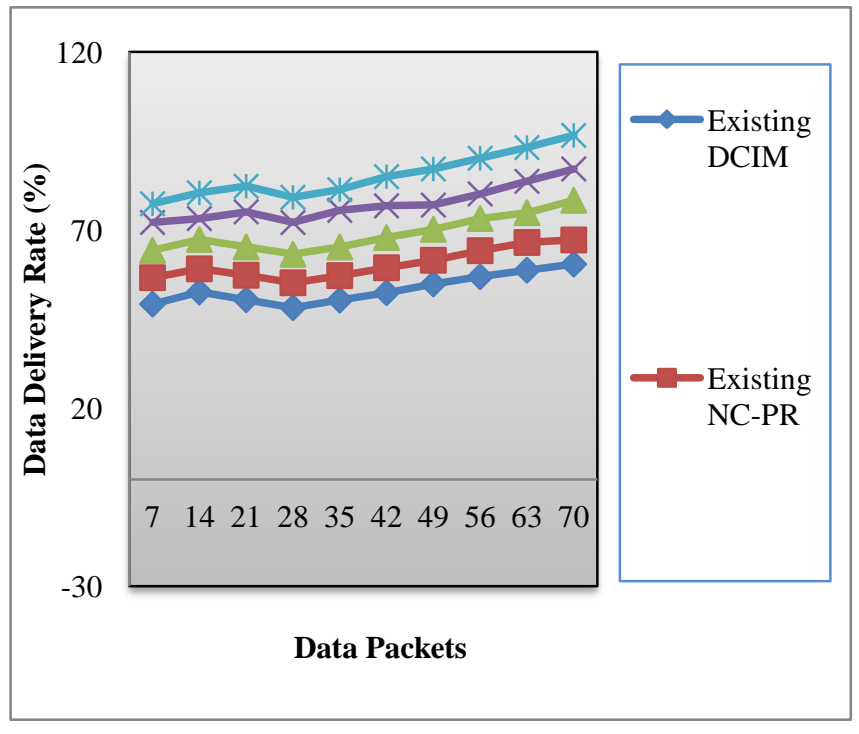

Fig. 4 Measure of data delivery rate

Fig.4. Illustrates the measure of data delivery rate using different methods such as proposed EO-NPCR, EE-PTRC, SQR-DD and existing DCIM method developed and NC-PR method. The SQR-DD method considerably increases the data delivery rate when compared to other proposed and existing methods. In SQR-DD method, communication history-based cooperative data forwarding evaluates the node cooperativeness factor before transmitting the data packet to the destination nodes. This Cooperative data forwarding algorithm is used to maximize the data delivery rate. Hence the data delivery rate using SQR-DD method is improved by $32 \%$ when compared to existing DCIM and NC-PR methods. Similarly, EE-PTRC framework increases the data delivery rate by $26 \%$ and EO-NPCR increases the data delivery rate by $17 \%$ compared to existing DCIM and NC-PR methods.

\section{Impact of Data Delivery Overhead}

The data delivery overhead is illustrated by the time taken to perform data communication over the mobile nodes with respect to the data size. The data delivery overhead is measured in the network as follows.

DDO $=$ DataCommunicationTime $*$ Date size

In the equation (4), the data delivery overhead ' $D D O$ ' is computed in terms of milliseconds (ms). If the data delivery overhead is low, then the method is said to be more efficient. 
An Enhanced Method of Secured Energy - Efficient Data Communication in MANET for Emergency Rescue Operation

Table. 4 Tabulation for Data Delivery Overhead

\begin{tabular}{|c|c|c|c|c|c|}
\hline \multirow{2}{*}{ Data size (KB) } & \multicolumn{5}{|c|}{ Data Delivery Overhead (ms) } \\
\cline { 2 - 6 } & $\begin{array}{c}\text { Existing } \\
\text { DCIM }\end{array}$ & $\begin{array}{c}\text { Existing } \\
\text { NC-PR }\end{array}$ & $\begin{array}{c}\text { Proposed } \\
\text { EO-NPCR }\end{array}$ & $\begin{array}{c}\text { Proposed } \\
\text { EE-PTRC }\end{array}$ & $\begin{array}{c}\text { Proposed } \\
\text { SQR-DD }\end{array}$ \\
\hline 10 & 12.4 & 13.5 & 11.4 & 6.2 & 9.3 \\
\hline 20 & 17.3 & 19.3 & 14.8 & 10.3 & 12.4 \\
\hline 30 & 22.2 & 24.2 & 20.7 & 15.2 & 13.3 \\
\hline 40 & 20.4 & 22.4 & 17.9 & 18.8 & 20.9 \\
\hline 50 & 25.9 & 28.2 & 23.1 & 16.7 & 18.8 \\
\hline 60 & 25.6 & 28.3 & 22.2 & 23.6 & 26.3 \\
\hline 70 & 31.1 & 33.2 & 28.7 & 27.4 & 29.8 \\
\hline 80 & 34.3 & 36.3 & 31.3 & 28.2 & 30.5 \\
\hline 90 & 35.2 & 38.4 & 32.6 & 31.8 & 34.7 \\
\hline
\end{tabular}

Table 4 describes the data delivery overhead while data packet transmission using proposed EO-NPCR, EE-PTRC, SQR-DD methods and existing DCIM, NC-PR methods. Number of data size is taken as input and varied from the range of 10 to 100 for experimental purpose. Any increase in the number of data size, data delivery overhead is also increased for all methods. However, the EE-PTRC framework is significantly provided to reduce the data delivery overhead compared to other methods.

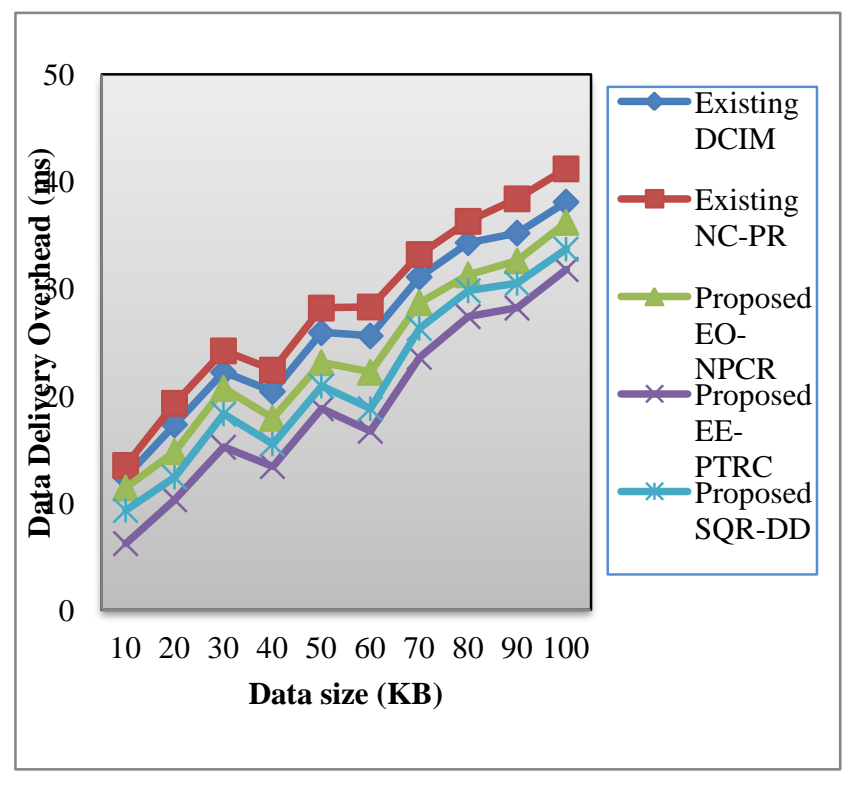

Fig. 5 Measure of data delivery overhead

Fig. 5.illustrates the measure of data delivery overhead using different methods such as proposed EO-NPCR, EEPTRC, SQR-DD and existing DCIM method and NC-PR method. The EE-PTRC framework considerably minimizes the data delivery overhead when compared to other proposed and existing methods. In EE-PTRC framework, polynomial time resource conserved channelized model is designed for choosing the data packet based on the cost with respect to the time. Therefore, the data delivery overhead is minimized in an optimized manner in emergency rescue application. Hence the data delivery overhead using EEPTRC is reduced by $35 \%$ compared to existing DCIM and NC-PR methods. Similarly, SQR-DD method reduces data delivery overhead by $29 \%$ and EO-NPCR reduces data delivery overhead by $12 \%$ compared to existing DCIM and NC-PR methods.

\section{E. Impact of Routing Delay}

Routing delay is calculated as sum of transmission delay, propagation delay and processing delay for data packet to be transmitted from source to destination node. Routing delay is mathematically formulated as follows.

Routing $_{D}=$ Transmission $_{D}+$ Propagation $_{D}+$ Processing $_{D}(5)$

In the equation (5), routing delay Routing $_{D}$ is measured in terms of milliseconds(ms). The routing delay contains the transmission delay 'Transmission ${ }_{D}$ ', propagation delay 'Propagation ${ }_{D}$ ' and processing delay 'Processing ${ }_{D}$ '. If the routing delay is low, then the method is will be more efficient. 
Table 5 describes the routing delay while data packet transmission using proposed EO-NPCR, EE-PTRC, SQRDD methods and existing DCIM, NC-PR methods. Number of mobile node density is varied from the range of 10 to 100 for experimental purpose. Table 5 shows that, for increasing in the number of mobile nodes, routing delay is also increased for all methods. However, the SQR-DD method significantly reducing the routing delays compared to other methods.

Table. 5 Tabulation for routing delay

\begin{tabular}{|c|c|c|c|c|c|}
\hline \multirow{2}{*}{$\begin{array}{c}\text { Mobile Node } \\
\text { Density }\end{array}$} & \multicolumn{4}{|c|}{ Routing Delay (ms) } \\
\cline { 2 - 6 } & $\begin{array}{c}\text { Existing } \\
\text { DCIM }\end{array}$ & $\begin{array}{c}\text { Existing } \\
\text { NC-PR }\end{array}$ & $\begin{array}{c}\text { Proposed } \\
\text { EO-NPCR }\end{array}$ & $\begin{array}{c}\text { Proposed } \\
\text { EE-PTRC }\end{array}$ & $\begin{array}{c}\text { Proposed } \\
\text { SQR-DD }\end{array}$ \\
\hline 10 & 2.56 & 2.15 & 1.93 & 1.52 & 1.4 \\
\hline 20 & 3.72 & 3.29 & 3.05 & 2.69 & 2.31 \\
\hline 30 & 5.22 & 4.68 & 4.28 & 3.81 & 3.33 \\
\hline 40 & 3.98 & 3.65 & 3.15 & 2.77 & 2.49 \\
\hline 50 & 6.04 & 5.48 & 5.11 & 4.53 & 4.08 \\
\hline 60 & 6.41 & 5.83 & 5.26 & 4.69 & 4.21 \\
\hline 70 & 9.85 & 9.15 & 8.53 & 7.88 & 6.92 \\
\hline 80 & 11.59 & 11.08 & 10.59 & 10.01 & 9.32 \\
\hline 90 & 12.03 & 11.39 & 10.97 & 10.36 & 10.02 \\
\hline 100 & 13.14 & 12.67 & 12.34 & 11.95 & 11.32 \\
\hline
\end{tabular}

Fig. 6.illustrates the measure of routing delay using different methods such as proposed EO-NPCR, EE-PTRC, SQR-DD and existing DCIM method and NC-PR method.

From Fig.6, it is clear that proposed SQR-DD method considerably minimizes the data delivery overhead when compared to other proposed and existing methods. In SQRDD method, better cooperative intermediate nodes are detected for reducing routing delay. Therefore, routing delay is minimized in an optimized manner in emergency rescue application.

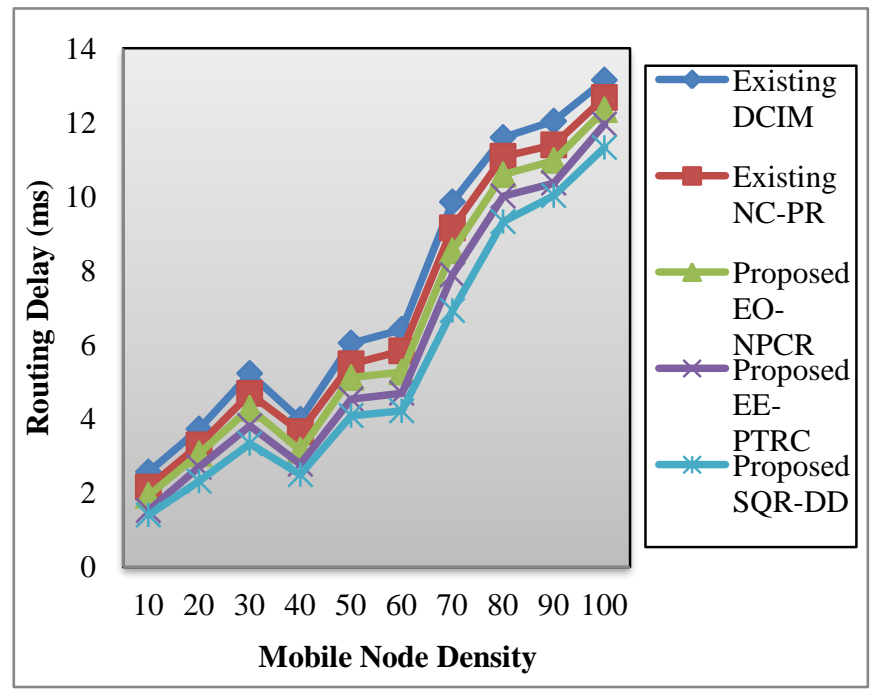

Fig. 6 Measure of routing delay

Hence the routing delay using SQR-DD method is reduced by $41 \%$ when compared to existing DCIM and NCPR methods. Similarly, EE-PTRC framework reduces routing delay by $32 \%$ and proposed EO-NPCR reduces routing delay by $14 \%$ compared to existing DCIM and NCPR methods.

\section{F. Impact of Bandwidth Consumption}

Bandwidth consumption is defined as the ratio of the bandwidth consumed for the data delivery rate to the total bandwidth available. The mathematical formulation for bandwidth utilization is written as given below.

$$
B=\frac{B_{C}}{B_{\text {avail }}}
$$

From equation (6), bandwidth ' $\mathrm{B}$ ' is measured with ' $B_{c}$ ' consumed bandwidth and ' $B_{\text {avail }}$ ', total available bandwidth. If the bandwidth consumption is low, then the method will be more effective. 
An Enhanced Method of Secured Energy - Efficient Data Communication in MANET for Emergency Rescue Operation

Table. 6 Tabulation for bandwidth consumption

\begin{tabular}{|c|c|c|c|c|c|}
\hline \multirow{2}{*}{$\begin{array}{c}\text { Mobile } \\
\text { Node (MN) }\end{array}$} & \multicolumn{5}{|c|}{ Bandwidth Consumption (Kbps) } \\
\cline { 2 - 6 } & $\begin{array}{c}\text { Existing } \\
\text { DCIM }\end{array}$ & $\begin{array}{c}\text { Existing } \\
\text { NC-PR }\end{array}$ & $\begin{array}{c}\text { Proposed } \\
\text { EO-NPCR }\end{array}$ & $\begin{array}{c}\text { Proposed } \\
\text { EE-PTRC }\end{array}$ & $\begin{array}{c}\text { Proposed } \\
\text { SQR-DD }\end{array}$ \\
\hline 10 & 4.42 & 3.98 & 3.12 & 2.16 & 2.51 \\
\hline 20 & 4.51 & 4.13 & 3.45 & 2.34 & 2.95 \\
\hline 30 & 5.56 & 4.89 & 3.97 & 2.78 & 3.47 \\
\hline 40 & 6.51 & 5.74 & 4.56 & 3.74 & 4.21 \\
\hline 50 & 6.94 & 6.12 & 5.21 & 4.11 & 4.87 \\
\hline 60 & 6.99 & 6.38 & 5.78 & 4.75 & 5.11 \\
\hline 70 & 7.42 & 6.87 & 6.23 & 4.98 & 5.46 \\
\hline 80 & 7.74 & 7.22 & 6.47 & 5.12 & 5.87 \\
\hline 90 & 8.14 & 7.86 & 7.32 & 5.67 & 6.21 \\
\hline 100 & 8.54 & 8.14 & 7.56 & 5.86 & 6.73 \\
\hline
\end{tabular}

Table 6 describes the bandwidth consumption based on the number of mobile nodes using proposed EO-NPCR, EEPTRC, SQR-DD methods and existing DCIM, NC-PR methods. Number of mobile node is varied from the range of 10 to 100 , for increasing in the number of mobile nodes, bandwidth consumption are also increased using all the techniques. However, the EE-PTRC framework provides minimum bandwidth consumption compared to other methods.

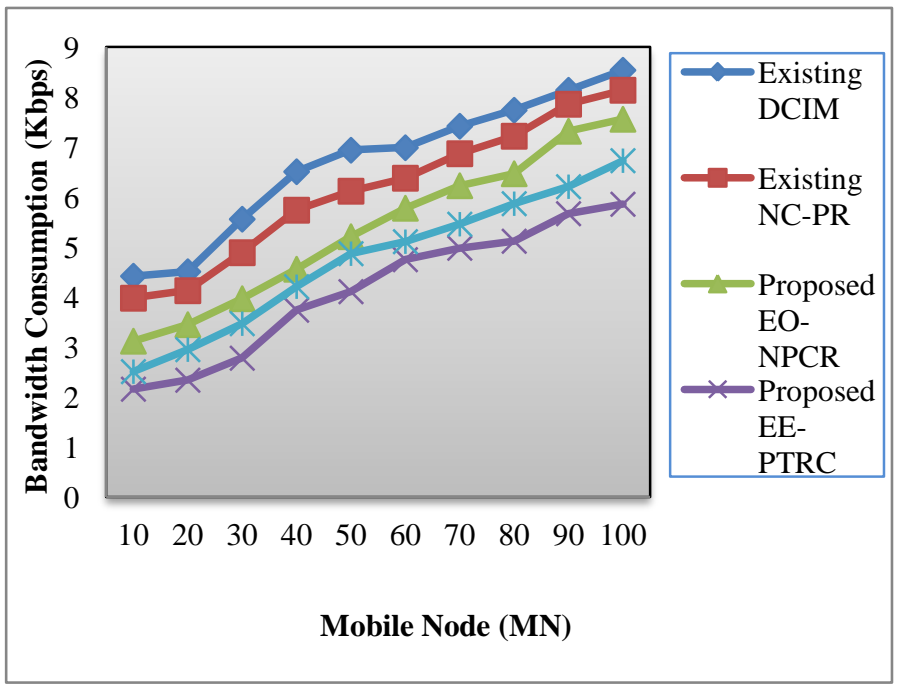

Fig. 7 Measure of bandwidth consumption

Fig.7. illustrates the measure of bandwidth consumption using different methods such as proposed EO-NPCR, EEPTRC, SQR-DD and existing DCIM method and NC-PR method, The EE-PTRC framework considerably reduces the bandwidth consumption compared to other proposed and existing methods. This reduction achieved by using Two Hop Propagate Bandwidth Allocation model in emergency rescue application. This model evaluates the number of bandwidth consumed for different mobile nodes and distributing the node with data packets based on the minimum channel weight. Hence the bandwidth consumption in MANETs using proposed method EE-PTRC is reduced by $43 \%$ compared to existing DCIM and NC-PR methods. Similarly, SQR-DD method reduces bandwidth consumption by $35 \%$ and EO-NPCR reduces bandwidth consumption by $22 \%$ compared to existing DCIM and NCPR methods.

\section{CONCLUSION}

A perfect illustration is discussed on the analysis of proposed EO-NPCR, EE-PTRC, SQR-DD methods with

existing Distributed Cache Invalidation Method (DCIM) and namely Neighbour Coverage-based Probabilistic Rebroadcast (NC-PR) method and provides effective emergency rescue application. Theoretical analysis and experimental result show that, routing energy consumption using proposed EO-NPCR is reduced by detecting maximum likelihood path for transferring the nodes from source to destination. Then, clustering accuracy is improved in EO-NPCR with the help of partitioned cluster routing algorithm. Data delivery overhead is reduced in the EEPTRC framework by selecting the data packet based on the cost with respect to the time. This method also reduces the bandwidth consumption with the aid of two hop propagate bandwidth allocation model. Routing delay in SQR-DD method is reduced by discovering better cooperative intermediate nodes. In addition, SQR-DD method effectively improves the data delivery rate by calculating the node cooperativeness factor in emergency rescue application.

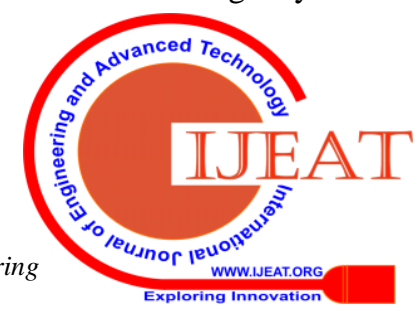




\section{REFERENCES}

1. Dipayan Bose, Arnab Banerjee, Aniruddha Bhattacharyya, Himadri Nath Saha, Debika Bhattacharyya \& Banerjee, PK 2012, 'An Efficient Approach to Secure Routing in MANET', Advances in Computing and Information Technology, Springer, vol. 176, pp. 765-776.

2. Dusit Niyato, Ekram Hossain \& Ping Wang 2011, 'Optimal Channel Access Management with QoS Support for Cognitive Vehicular Networks', IEEE Transactions on Mobile Computing, vol. 10, no. 4,pp. 573-591.

3. Eleftheria Athanasopoulou, Loc X Bui, Tianxiong Ji, Srikant, R \& Alexander Stolyar 2013, 'Back-Pressure-Based Packet-by-Packet Adaptive Routing in Communication Networks', IEEE/ACM Transactions on Networking, vol. 21, no. 1, pp. 244-257.

4. Filomena de Santis \& Delfina Malandrino 2014, 'QoS-Based Web Service Discovery in Mobile Ad Hoc Networks using Swarm Strategies', Hindawi Publishing Corporation, Journal of Computer Networks and Communications, vol. 2014, pp. 1-13.

5. Gautam M Borkar \& Mahajan, AR 2016, 'A secure and trust based on demand multipath routing scheme for self-organized mobile ad-hoc networks', Wireless Network, Springer, pp. 1-18.

6. Harold Robinson, Y \& Rajaram, M 2015 'Energy-Aware Multipath Routing Scheme Based on Particle Swarm Optimization in Mobile AdHoc Networks', Hindawi Publishing Corporation, The Scientific World Journal, pp. 1-9.

7. Hong $\mathrm{Xu} \&$ Baochun Li 2013, 'Resource Allocation with Flexible Channel Cooperation in Cognitive Radio Networks', IEEE Transactions on Mobile Computing, vol. 12, no. 5, pp. 957-970.

8. Javad Vazifehdan, Venkatesha Prasad, R \& Ignas Niemegeers 2014, 'Energy-efficient Reliable Routing Considering Residual Energy in Wireless Ad Hoc Networks', IEEE Transactions on Mobile Computing, vol. 13, no. 2, pp. 434-447.

9. Jing Dong, Reza Curtmola \& Cristina Nita-Rotaru 2011, 'Secure HighThroughput Multicast Routing in Wireless Mesh Networks', IEEE Transactions on Mobile Computing, vol. 10, no. 5, pp. 653-668

10. Jinhua Zhu \& Xin Wang 2011, 'Model and Protocol for Energy efficient Routing over Mobile Ad Hoc Networks', IEEE Transactions on Mobile Computing, vol. 10, no. 11, pp. 1546-1557.

11. J. Sathiamoorthy, B. Ramakrishnan b \& Usha. M 2017, ' Design of aproficient hybrid protocol for efficient route discovery and secure data transmission in CEAACK MANETs', Journal of Information Security and Applications, Elsevier, vol.36, pp.43-58.

12. Junbeom Hur \& Kyungtae Kang 2014, 'Secure Data Retrieval for Decentralized Disruption-Tolerant Military Networks', IEEE/ACM Transactions on Networking, vol. 22, no. 1, pp. 16-26.

13. Lajos Hanzo II \& Rahim Tafazolli 2011, 'QoS-Aware Routing and Admission Control in Shadow-Fading Environments for Multirate MANETs', IEEE Transactions on Mobile Computing, vol. 10, no. 5,pp. 622-637.157

14. Hussein I., Mkpojiogu E.O.C., Hussain A. (2019). A Focus Group Assessment Of Participants' UX Knowledge And Their Motivations For Participating In A UXD Community Of Practice. Journal of Advanced Research in Dynamical and Control Systems. Vol 11. No 5 Special Issue. Page 1496-1499

15. Neeraj Sharma, Chaurasia, BP \& Gupta, SK 2015, 'An Energy efficient Administration Based Secure Routing in MANET', International Journal of Emerging Technology and Advanced Engineering, vol. 5, no. 7 , pp. 358-364.158

16. Hussain, A., Manikanthan, S.V., Padmapriya, T. Nagalingam M. Wireless Networks (2019). https://doi.org/10.1007/s11276-01902121-4 Genetic algorithm based adaptive offloading for improving IoT device communication efficiency, Wireless Networks, 2019.

17. Sajal Sarkar \& Raja Datta 2016, 'A secure and energy-efficient stochastic multipath routing for self-organized mobile $\mathrm{Ad}$ Hocnetworks', Ad Hoc Networks, Elsevier, vol. 37, pp. 209-227.

\section{AUTHOR PROFILE}

Jayachitra. $\mathbf{S}$ is an Associate Professor, presently working in department of Electronics and Communication Engineering, Vivekanandha College of Engineering for Women, Tiruchengode. She completed her doctorate in the area of Mobile Ad-Hoc Networks in the year of 2018. She published nearly eight papers related to her research in reputed journals. She is a researcher in wireless communication.

Thiagarajan. $\mathrm{N}$ is Presently working as an Assistant Professor in the Department of Medical Electronics Engineering Sengunthar College of Engineering, Tiruchengode. He completed his Master Degree-Applied
Electronics in the year 2010. His area of interest include Digital Signal Processing, Digital Image Processing, Mobile Ad-Hoc Networks etc. He is expert of writing Mat Lab Coding. 